\title{
PRODUÇÃO DE CORDEIROS EM PASTAGEM DE AZEVÉM E TREVO-BRANCO SOB DIFERENTES NÍVEIS DE RESÍDUOS DE FORRAGEM ${ }^{1}$
}

\author{
MARCOS WEBER DO CANTO², EDUARDO LONDERO MOOJEN ${ }^{3}$, \\ PAULO CÉSAR DE FACCIO CARVALHO ${ }^{4}$ e JOSÉ HENRIQUE SOUZA DA SILVA ${ }^{5}$
}

\begin{abstract}
RESUMO - Na presente pesquisa, objetivou-se analisar os efeitos de diferentes níveis de resíduos de forragem na produção de cordeiros. O experimento foi estabelecido em Tupanciretã, região do Planalto Médio do Estado do Rio Grande do Sul, em uma pastagem de azevém cv. Estanzuela 284 (Lolium multiflorum Lam) + trevo-branco cv. Yi (Trifolium repens L.), submetida a diferentes níveis de resíduo de matéria seca (RMS). O delineamento experimental utilizado foi o completamente casualizado, estudando-se os níveis de RMS mantidos na pastagem de 1.119, 1.320, 1.477, 1.695, 2.146, 2.166, $2.410 \mathrm{e}$ $2.483 \mathrm{~kg}$ de matéria seca por hectare (MS/ha). O período experimental foi de 30/09 a 08/12/1992 e os animais utilizados foram cordeiros cruza Ile de France x Corriedale desmamados aos 80 dias. Foram avaliados o ganho de peso médio diário (GMD), ganho de peso vivo/ha (GPV/ha), carga animal, animais dia/ha e a eficiência de conversão de MS em $\mathrm{kg}$ de peso vivo de cordeiros. Os resultados obtidos mostraram uma relação linear positiva entre o GMD e GPV/ha com o aumento nos níveis de RMS/ha, observando-se nos níveis de RMS superiores a $2.400 \mathrm{~kg}$ um GMD de 0,12 kg/cordeiro/dia e um GPV/ha médio de $497 \mathrm{~kg}$. Não houve relação quanto à carga animal e animais.dia/ha; verificou-se uma relação linear negativa entre a eficiência de conversão de MS com os níveis de RMS estudados. Os resultados demonstram o efeito dos níveis de RMS na resposta animal.
\end{abstract}

Termos para indexação: ganho de peso médio diário, ganho de peso vivo, pastejo, resíduo de matéria seca.

\section{LAMB PRODUCTION IN A MIXTURE OF ITALIAN RYEGRASS AND WHITE CLOVER UNDER DIFFERENT FORAGE MASS LEVELS}

\begin{abstract}
The objective of this research was to analyse the effects of different forage mass levels on lamb production. The experiment was conducted in Tupanciretã, Planalto Médio region at the State of Rio Grande do Sul, Brazil, in a mixture of italian ryegrass cv. Estanzuela 284 (Lolium multiflorum Lam.) + white clover cv. Yi (Trifolium repens L.), under different forage mass (FM) levels. The experimental design used was completely randommized, and the FM levels studied were: 1,119, 1,320, 1,477, $1,695,2,146,2,166,2,410$ and $2,483 \mathrm{~kg} / \mathrm{ha}$ of dry matter (DM - kg/ha). The experimental period was from September 30 to December 8, 1992 and the animals used were cross lamb Ile de France x Corriedale early weaned (mean \pm 80 days). Were evaluated the daily liveweight gain (DLG), gain per hectare (G/ha), stocking rate carryed, animals.day/ha and the DM of conversion efficience in $\mathrm{kg}$ of lamb liveweight. The results showed a positive linear relationship between DLG and G/ha with increased of FM levels. The FM levels higher to $2,400 \mathrm{~kg}$ of DM/ha reached $497 \mathrm{~kg}$ of total lamb liveweight gain/ha, and DLG/lamb of $0.12 \mathrm{~kg} /$ day. There was no FM levels effect at the stocking rate and animals.day/ha; there was a negative linear relationship between DM of conversion efficience with FM levels studied. The results show the effect of FM levels on animal response.
\end{abstract}

Index terms: average daily gain, gain per hectare, grazing, forage mass.

\footnotetext{
${ }^{1}$ Aceito para publicação em 29 de maio de 1998. Extraído da Dissertação de Mestrado apresentada pelo primeiro autor à UFSM, Santa Maria, RS.

${ }^{2}$ Eng. Agr., M.Sc., Prof. Assistente, Dep. de Zootecnia, Universidade Estadual de Maringá, Av. Colombo, 5790, CEP 87020-900 Maringá, PR. E-mail: dzo@dfi.uem.br
}

\footnotetext{
${ }^{3}$ Eng. Agr., Dr., Prof. Titular, Dep. de Zootecnia, UFSM, CEP 97119-900 Santa Maria, RS.

${ }^{4}$ Zoot., Dr., Prof. Adjunto, Dep. de Agrometeorologia e Plantas Forrageiras, UFRGS, CEP 90001-970 Porto Alegre, RS.

${ }^{5}$ Eng. Agr., Ph.D, Prof. Adjunto, Dep. de Zootecnia, UFSM.
} 


\section{INTRODUÇÃO}

Os rebanhos ovinos na Região Sul do Brasil são criados quase que exclusivamente em campo nativo, o que determina baixos níveis de produtividade, principalmente pelo fato de as pastagens naturais apresentarem produção tipicamente estacional.

O baixo valor nutritivo dessas pastagens, associado ao nível reduzido de oferta de forragem durante os meses de outono-inverno, afeta o desempenho dos bovinos sob pastejo, conforme foi observado por Moojen (1991).

Com cordeiros sob pastejo em campo nativo, também são baixos os ganhos de peso/animal, conforme relatado por Siqueira et al. (1984). Por essa razão, a utilização de pastagens cultivadas de inverno constitui-se em uma alternativa para suprir o período de carência alimentar.

Experimentos recentes com bovinos têm mostrado o potencial de gramíneas e leguminosas de inverno na obtenção de altos rendimentos por animal e por unidade de área (Quadros \& Maraschin, 1987 e Moraes, 1991). Ao mesmo tempo, em ecossistemas de pastagens consorciadas de inverno sob pastejo, o nível de oferta de forragem é fator fundamental para o manejo e utilização dessas pastagens, por seu efeito no desempenho do animal e na produção do produto animal por área. Verifica-se na literatura que diversos trabalhos corroboram essa teoria.

Com respeito às condições do Rio Grande do Sul, porém, existem poucos estudos sobre o uso e manejo de pastagens consorciadas de inverno para ovinos. Assim, o presente trabalho teve por objetivo estudar os efeitos de níveis de resíduos de matéria seca (RMS) na produção de cordeiros.

\section{MATERIAL E MÉTODOS}

O experimento foi desenvolvido na Fazenda do Espinilho, no município de Tupanciretã, região do Planalto Médio do Rio Grande do Sul, no período de 30/09 a 08/12/1992. O solo constituiu-se em um Latossolo Roxo, cuja análise apresentou os seguintes resultados: $\mathrm{pH}=5,7$ (1:1 em água), $4,0 \mathrm{mg} / \mathrm{dm}^{3}$ de fósforo, $138 \mathrm{mg} / \mathrm{dm}^{3}$ de potássio, 59,3 $\mathrm{g} / \mathrm{kg}$ de matéria orgânica, 0 e $9,61 \mathrm{cmol}_{\mathrm{c}} / \mathrm{L} \mathrm{de}$ $\mathrm{Al}^{3+} \mathrm{e} \mathrm{Ca}^{2+}+\mathrm{Mg}^{2+}$, respectivamente.

A pastagem de aveia-preta (Avena strigosa $\mathrm{Schreb})+$ azevém + trevo-branco foi estabelecida por meio de uma aração e duas gradagens, seguida de uma adubação com $600 \mathrm{~kg} / \mathrm{ha}$ da fórmula 0-25-20; a semeadura realizou-se em abril de 1992. A densidade de semeadura foi de $75 \mathrm{~kg} / \mathrm{ha} \mathrm{de}$ aveia-preta, $25 \mathrm{~kg} / \mathrm{ha}$ de azevém e $6 \mathrm{~kg} / \mathrm{ha}$ de trevo-branco + inoculante. O experimento ocupou uma área de 1,5 ha, subdividida em quatro piquetes de 0,225 ha e quatro de 0,15 ha. Embora a aveia-preta tenha sido semeada no início do experimento, verificou-se uma reduzida participacão percentual na composição botânica desta espécie.

O delineamento experimental utilizado foi o completamente casualizado, com duas repetições, e os tratamentos comparados eram quatro níveis de RMS; de 600, 1.200, 1.800 e $2.400 \mathrm{~kg} / \mathrm{ha}$ de MS. No entanto, a demora na estimativa do percentual de MS da pastagem, entre outras razões, determinou uma variação do resíduo mantido nas parcelas experimentais em relação aos resíduos pretendidos. Por isso, os RMS estimados em cada parcela experimental foram de 1.119 para a repetição um do RMS pretendido de $600 \mathrm{~kg} / \mathrm{ha}$ (T600R1), 1.320 (T600R2), 1.477 (T1200R1), 1.695 (T1200R2), 2.146 (T1800R1), 2.166 (T1800R2), 2.410 (T2400R1) e 2.483 (T2400R2) kg/ha de MS.

Os animais utilizados foram cordeiros oriundos de cruzamentos das raças Ile de France x Corriedale, desmamados aos 80 dias e com peso médio inicial de $17,5 \mathrm{~kg}$. O sistema de pastejo adotado foi o contínuo, com ajuste da carga animal a cada 21 dias para manter os RMS próximos aos níveis desejados, de acordo com o método descrito por Mott \& Lucas (1952). Utilizaram-se cinco cordeiros testers por parcela experimental, com exceção das parcelas com RMS de 1.119 e $1.320 \mathrm{~kg} / \mathrm{ha}$ de MS, onde foram usados quatro, e no RMS de $1.477 \mathrm{~kg} / \mathrm{ha}$ de MS, com uso de três. Foram utilizados cordeiros inteiros como testers, e como reguladores, animais castrados e inteiros. Na fase inicial do experimento, em todas as unidades experimentais, os RMS encontravam-se em níveis superiores a $3.100 \mathrm{~kg} / \mathrm{ha} \mathrm{de} \mathrm{MS.}$ Foram então colocadas vacas em lactação com terneiros ao pé nas parcelas T $600 \mathrm{R}_{1}$ e $\mathrm{R}_{2}, \mathrm{~T} 1.200 \mathrm{R}_{1}$ e $\mathrm{R}_{2}$ e $\mathrm{T} 1.800 \mathrm{R}_{1}$ e $\mathrm{R}_{2}$, com a finalidade de se atingir os níveis de resíduos pretendidos. Entretanto, este manejo causou um excesso de pisoteio e grandes perdas de forragem nas parcelas com RMS de $1.119,1.320$ e $1.477 \mathrm{~kg} / \mathrm{ha}$ de MS.

Durante o período experimental, os animais tiveram acesso permanente a sal mineral e água, e foram pesados a cada 23 dias, sempre à tarde e sem jejum prévio. Foram vermifugados em 21/09/92 (Ivermectin), 19/10/92 (Xeron), 12/11/92 e 08/12/92 com Disofenol.

O RMS foi estimado pelo método da dupla-amostragem (Wilm et al., 1944), e o nível de RMS/ha calculado pela equação proposta por Gardner (1986). As taxas de acúmulo de MS foram estimadas por meio da técnica do triplo em- 
parelhamento (Moraes, 1991), sendo usadas duas gaiolas por unidade experimental, e a equação preconizada por Campbell (1966) permitiu o cálculo da taxa de acúmulo de MS.

A taxa de desaparecimento da pastagem baseou-se no método de Moraes (1991), proposto recentemente e descrito a seguir:

$$
D_{j}=\frac{T_{j}+\left(R_{i}-R_{f}\right)}{n_{j}}
$$

em que:

$D_{\mathrm{j}}=$ taxa de desaparecimento de MS em $\mathrm{kg} / \mathrm{ha} /$ dia no período j;

$\mathrm{T}_{\mathrm{j}}=$ taxa de acúmulo de MS no período $\mathrm{j}$;

$\mathrm{R}_{\mathrm{i}}=\mathrm{RMS}$ em $\mathrm{kg} / \mathrm{ha}$ no início do período j;

$\mathrm{R}_{\mathrm{f}}=\mathrm{RMS}$ em $\mathrm{kg} / \mathrm{ha}$ no final do período $\mathrm{j}$;

$\mathrm{n}_{\mathrm{j}}=$ número de dias do período $\mathrm{j}$.

Para o cálculo da produção de MS utilizou-se o RMS da fase inicial adicionado às taxas de acúmulo de MS estimadas ao longo do período experimental.

A pressão de pastejo (PP) foi obtida pela soma do RMS no final do período mais a taxa de acúmulo de MS multiplicada pelo número de dias do período; este valor foi dividido pela carga animal e multiplicado por 100 , expressando a PP real do período. O componente folhas verdes (lâmina verde + bainha verde) foi determinado a partir das três amostras colhidas por unidade experimental destinadas à separação botânica. Essas foram secas em estufa, e por meio do peso seco do componente folhas verdes calculou-se a participação percentual deste componente no período experimental. A percentagem de folhas verdes na MS, multiplicada pelo RMS do período, permitiu estimar a MS de folhas verdes.

Determinou-se a participação percentual de aveia, azevém e trevo-branco a partir de três amostras colhidas aleatoriamente na unidade experimental, no momento em que realizou-se a dupla amostragem da pastagem. As amostras da separação botânica foram secadas em estufa arforçado e, com base no peso seco de cada espécie, foi obtida a composição botânica da pastagem. A contribuição da aveia foi observada somente na primeira amostragem da pastagem, e nas posteriores não foi observada a participação da aveia nas unidades experimentais.

O percentual de digestibilidade in vitro da matéria orgânica (DIVMO) foi estimado pelo método de Tilley \& Terry (1963), com modificações feitas por Pires et al. (1979). O teor de proteína bruta (PB) foi calculado a partir do teor de $\mathrm{N}$ da forragem e multiplicado por 6,25 , sendo o teor de $\mathrm{N}$ das plantas determinado pelo método semimicro de Kjeldhal (Association of Official Agricultural Chemists, 1984).
A eficiência de conversão da MS foi obtida pela divisão da produção de MS/ha pelo GPV/ha, do período experimental, e a percentagem de utilização da pastagem foi calculada pela equação descrita por Gibb \& Treacher (1980).

O ganho médio diário (GMD) dos cordeiros testers foi estimado subtraindo-se do peso médio final o peso inicial do período experimental e dividindo-o pelo número de dias em que os cordeiros permaneceram na pastagem. A determinação do número de animais.dia/ha foi obtida pelo número de animais mantidos na parcela experimental em cada dia, multiplicado pelo número de dias que permaneceram na parcela.

$\mathrm{O} \mathrm{GPV} / \mathrm{ha}$ foi estimado pelo número de animais.dia/ha multiplicado pelo GMD dos cordeiros testers. A carga animal foi calculada com o peso vivo médio dos cordeiros em cada período, e este peso, após, foi dividido pela área da unidade experimental, sendo expresso em $\mathrm{kg} / \mathrm{ha}$ de peso vivo.

Foram analisadas as variáveis GPV/ha, PP, DIVMO, participação do azevém, GMD, utilização da pastagem, percentual de trevo-branco e PB, animais.dia/ha e carga animal, por meio de modelos de regressão polinomial em função dos RMS observados, com o auxílio do programa SAS (SAS Institute, 1985).

\section{RESULTADOS E DISCUSSÃO}

A Tabela 1 mostra a relação das variáveis dependentes com os níveis de RMS/ha estudados, que apresentaram significância quando foram testados os modelos linear e quadrático. Mostra também a relação entre o GMD dos cordeiros com os níveis de MS de folhas verdes/ha da pastagem. Constata-se que a relação das variáveis GPV/ha, PP, DIVMO, participação percentual de azevém e GMD com os níveis de RMS/ha avaliados foi linear e positiva, com níveis de significância de $(\mathrm{P}<0,0008),(\mathrm{P}<0,0006)$, $(\mathrm{P}<0,0025),(\mathrm{P}<0,0293)$ e $(\mathrm{P}<0,00001)$, respectivamente. Observa-se, também, que houve uma relação linear e positiva $(\mathrm{P}<0,0045)$ entre o GMD dos cordeiros com os níveis de MS de folhas verdes/ha da pastagem. À medida que o nível de resíduo de forragem se elevou na pastagem, foi obtido um maior rendimento em $\mathrm{kg} / \mathrm{ha}$ de peso vivo de cordeiro, menor PP, aumentos na DIVMO, maior participação percentual de azevém e maior GMD dos cordeiros. Com relação às variáveis percentagem de utilização da pastagem e eficiência de conversão da MS, verificou-se uma relação linear e negativa, como mostra a 
Tabela 1, com níveis de significância de $(\mathrm{P}<0,0029)$ e $(\mathrm{P}<0,0096)$, respectivamente. Com o aumento do nível de RMS/ha ocorreu uma diminuição na percentagem de utilização da pastagem e melhorou a eficiência de conversão da MS em produto animal/ha.

Os resultados referentes aos GPV/ha são uma decorrência, em parte, do manejo feito para baixar os resíduos na fase inicial do experimento. Conforme exposto anteriormente, foram utilizadas vacas para se atingir os níveis de RMS requeridos em cada tratamento. Como conseqüência, maior foi o número de vacas utilizado nos baixos RMS, em proporção à área de cada parcela experimental. Com isso, nas parcelas com RMS inferiores a $2.400 \mathrm{~kg} / \mathrm{ha}$, o nível de MS/ha foi rebaixado utilizando cordeiros e vacas, e estas não foram computadas no número de animais.dia/ha, ao passo que nos RMS superiores a $2.400 \mathrm{~kg} / \mathrm{ha}$ de MS foram utilizados somente cordeiros, acrescentados ao número de animais.dia/ha. Pode-se afirmar, assim, que a estimativa da produção animal, em $\mathrm{kg} / \mathrm{ha}$, das unidades experimentais com resíduos inferiores a $2.200 \mathrm{~kg} / \mathrm{ha}$ de MS teria atingido maiores valores aos aqui relatados.

Moraes (1991), trabalhando com novilhos em pastagens de pangola (Digitaria decumbens Stent), azevém e trevo-branco, no período do verão, verificou uma relação curvilinear quadrática quando relacionou o ganho por área à PP e ao resíduo. Não obstante, os estudos de Orr et al. (1990) e Penning et al. (1991) em pastagens temperadas, utilizando ovelhas com cordeiros ao pé, observaram que o maior rendimento de cordeiros ocorreu nos mais baixos RMS, porém à custa de uma reduzida taxa de crescimento dos cordeiros.
As estimativas obtidas nos níveis de RMS acima de $1.600 \mathrm{~kg}$ de $\mathrm{MS} / \mathrm{ha}$, comparadas ao resultado de GPV/ha verificado por Figueiró (1979) com cordeiros, na mistura de azevém e trevo-branco são superiores. Fazendo-se uma análise comparativa com outros estudos de pastejo com espécies de estação fria, realizados no Rio Grande do Sul, pode-se afirmar que os ganhos de produto animal por área (cerca de $500 \mathrm{~kg}$ no RMS de $2.400 \mathrm{~kg} / \mathrm{ha}$ de MS) foram muito bons, devido principalmente ao curto período experimental (70 dias) e, também, pelo fato de a pastagem estar finalizando o período de produção.

A PP variou de 5,98 a 13,18 kg de MS/100 kg de peso vivo/dia.

Com a quantidade de alimento em oferta em uma pastagem sendo regulada pelo controle do nível de RMS, tem-se como conseqüência PP mais baixa nos altos resíduos de forragem. Efeito análogo foi mostrado no experimento realizado por Moojen (1991) em campo nativo.

A DIVMO decresceu com a redução no nível de MS/ha, e os valores de DIVMO oscilaram de $53,1 \%$ a $41,4 \%$.

No trabalho conduzido por Moojen (1991) foi observado que a resposta da DIVMO à elevação do nível de RMS é linear e negativa. O rebaixamento do nível de MS/ha feito na fase inicial do experimento determinou um aumento na participação dos caules na estrutura das plantas dos mais baixos RMS, o que por sua vez determinou a resposta verificada entre DIVMO e RMS no presente experimento. Os baixos teores de DIVMO encontrados podem ser atribuídos à alta participação dos caules na estrutura das plantas. Convém salientar que os valores de DIVMO

TABELA 1. Relação linear dos diferentes níveis de resíduo de matéria seca com as variáveis dependentes.

\begin{tabular}{|c|c|c|c|}
\hline Variável dependente $^{1}$ & Equação & $\mathrm{R}^{2}$ & $\mathrm{P}(\mathrm{P}<)$ \\
\hline GPV (kg/ha) & $Y=65,11+0,18 X$ & 0,86 & 0,0008 \\
\hline $\mathrm{PP}$ (kg MS/100 kg PV/dia) & $Y=2,33+0,004 X$ & 0,87 & 0,0006 \\
\hline DIVMO $(\%)$ & $Y=31,74+0,0086 X$ & 0,81 & 0,0025 \\
\hline Azevém (\%) & $Y=24,39+0,017 X$ & 0,57 & 0,0293 \\
\hline GMD (kg/cordeiro/dia) & $Y=-0,015+0,000055 X$ & 0,96 & 0,00001 \\
\hline GMD c/ MS folhas verdes & $Y=-0,024+0,00015 X$ & 0,76 & 0,0045 \\
\hline Utilização da pastagem (\%) & $Y=82,26-0,016 X$ & 0,80 & 0,0029 \\
\hline 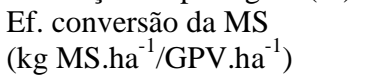 & $Y=37,80-0,01 X$ & 0,70 & 0,0096 \\
\hline
\end{tabular}

${ }^{1}$ GPV: ganho de peso vivo; PP: pressão de pastejo; DIVMO: digestibilidade in vitro da matéria orgânica; GMD: ganho médio diário; MS: matéria seca. 
foram obtidos de plantas cortadas no nível do solo, e não correspondem à forragem que o animal ingere em condições de pastejo.

A participação do azevém nos diferentes níveis de RMS oscilou de $41,3 \%$ a $70,9 \%$, e sua redução nos níveis baixos de RMS foi conseqüência do aumento na proporção de material morto. Neste trabalho, no momento da separação botânica descartouse das plantas de azevém e trevo-branco a fração senescente.

O GMD dos cordeiros na pastagem de azevém e trevo-branco submetida a distintos resíduos oscilou de 0,051 a $0,122 \mathrm{~kg} / \mathrm{animal} / \mathrm{dia}$.

Nos diversos trabalhos nos quais o objeto de estudo é a relação nível de RMS:desempenho por animal, tem-se encontrado uma relação curvilinear demonstrada nos estudos realizados por Maxwell (1983) e Sheath et al. (1987). Todavia, neste experimento, foi constatado um modelo linear entre as variáveis GMD e nível de RMS, que pode ser explicado por não ter-se trabalhado com RMS superiores a $2.500 \mathrm{~kg} / \mathrm{ha}$ de MS, quando possivelmente se obteria uma relação curvilinear. Tal consideração pode ser confirmada pelos resultados de Gibb \& Treacher (1976) com cordeiros desmamados, e de Penning et al. (1986) com cordeiros ao pé da mãe, nos quais constataram-se relações curvilineares entre o GMD dos cordeiros e as disponibilidades de forragem estudadas. Os resultados obtidos, em termos de GMD dos cordeiros, comprovam as afirmações de Rattray et al. (1987) de que a relação entre o GMD e o RMS em níveis de até $2.200 \mathrm{~kg}$ de MS/ha é linear e positiva. O efeito da menor PP (Tabela 1) e da qualidade de forragem (DIVMO e PB) nos mais altos resíduos determinaram a resposta linear do GMD dos cordeiros com o aumento do RMS.

O GMD oscilou de $0,051 \mathrm{~kg} /$ animal $/$ dia no RMS com $604 \mathrm{~kg} / \mathrm{ha}$ de MS de folhas verdes a $0,122 \mathrm{~kg} /$ animal $/$ dia no RMS com $906 \mathrm{~kg} / \mathrm{ha}$ de MS de folhas verdes.

Conforme Sheath et al. (1987), nos animais sob pastejo, o maior fator determinante do consumo é a MS de folhas verdes. Com base neste efeito, Burns et al. (1989) enfatizam a necessidade de se realizar tais estimativas em todos os experimentos de pastejo. No presente experimento, não houve efeito significativo das variáveis carga animal e taxa de desapare- cimento da pastagem com o RMS. Todavia, o GMD dos cordeiros variou nos diferentes tratamentos. Isto pode ser explicado pelo consumo de forragem com qualidade mais alta e pastejo seletivo proporcionado principalmente pela maior oferta de folhas verdes nos maiores RMS.

Analisando-se o resultado mostrado na Tabela 1, verifica-se que ele é similar ao encontrado por Hendricksen \& Minson (1980), com vacas Jersey em pastagens de Lablab purpureus cv. Rongai. Neste trabalho, o consumo de forragem, expresso com base na matéria orgânica, relacionou-se significativamente com o rendimento de MS de folhas verdes. Os autores destacam que a preferência por folhas é, provavelmente, em virtude do menor gasto de energia requerido para cortar as folhas em relação aos caules amadurecidos, que exigem esforços adicionais na mastigação.

Segundo Gibb \& Treacher (1976) e Penning et al. (1991), sob condições de pastejo contínuo, devem ser feitas algumas ressalvas em pastagens manejadas com altos RMS objetivando um alto GMD, uma vez que, em tais condições, ocorre uma deterioração mais rápida da estrutura da pastagem, reduzindo a relação folha/caule. Em decorrência desse efeito nem sempre o GMD de cordeiros seria elevado.

Os GMD obtidos em RMS próximos a $2.500 \mathrm{~kg}$ de $\mathrm{MS} / \mathrm{ha}$ indicam a perspectiva de que cordeiros desmamados aos 80 dias e oriundos de sistemas de produção tradicionais sejam abatidos ainda com idade jovem, se colocados em pastagens cultivadas de verão com bons níveis de oferta de forragem. Moraes (1991), trabalhando com bovinos no verão, em capim pangola, relatou um bom GMD no período. O GMD nos mais altos resíduos foram próximos e inferiores aos observados por Gibb \& Treacher (1976) em azevém perene (Lolium perenne L.) e trevo-vermelho (Trifolium pratense L.), com cordeiros desmamados precocemente.

Na Tabela 1, observa-se que o percentual de utilização da pastagem oscilou de $63,2 \%$ no resíduo com $1.119 \mathrm{~kg} / \mathrm{ha}$ de MS a 38,4\% no RMS com $2.410 \mathrm{~kg} / \mathrm{ha}$ de MS.

Os resultados observados quanto ao percentual de utilização da pastagem, próximos a 40\%, nos RMS superiores a $2.400 \mathrm{~kg}$ de $\mathrm{MS} / \mathrm{ha}$, assemelham-se à recomendação feita por Geenty (1983), de 30\%, em práticas de manejo de pastagens para cordeiros. 
A eficiência de conversão da MS foi de $28,17 \mathrm{~kg}$ de MS $/ \mathrm{kg}$ de peso vivo no RMS com $1.320 \mathrm{~kg}$ de $\mathrm{MS} / \mathrm{ha}$ a $10,50 \mathrm{~kg}$ de $\mathrm{MS} / \mathrm{kg}$ de peso vivo no RMS com $2.410 \mathrm{~kg}$ de $\mathrm{MS} / \mathrm{ha}$.

Pela análise da Tabela 1, depreende-se que cordeiros em situações de pastejo, e em altos RMS/ha, necessitam de menores quantidades de MS para serem convertidas em produto animal. Segundo Blaser (1982), em situações de reduzido consumo de energia e baixo desempenho por animal, grande parte da matéria seca digestível ingerida é utilizada na mantença do peso corporal, o que determina menor eficiência de conversão de MS em produto animal. Moraes (1991) citou efeito semelhante quando condicionou uma pastagem de pangola, azevém e trevo-branco pastejada por novilhos a diferentes PP.

Os resultados médios apresentados na Tabela 2 mostram a taxa de desaparecimento da pastagem, percentagem de trevo-branco e $\mathrm{PB}$, número de animais.dia/ha e a carga animal mantida na pastagem. Essas variáveis não apresentaram relação significativa com os tratamentos estudados, quando os modelos linear e quadrático foram testados.

A taxa de desaparecimento variou de 52,9 a $104,0 \mathrm{~kg} / \mathrm{ha} / \mathrm{dia}$ de MS, sendo estimada em média uma taxa de desaparecimento da pastagem de $82,1 \mathrm{~kg}$ de $\mathrm{MS} / \mathrm{ha} /$ dia de acordo com a Tabela 2.

A não-significância entre a taxa de desaparecimento da pastagem e os RMS deveu-se ao fato de não ter havido diferença na carga animal utilizada nas diferentes parcelas experimentais. A taxa de desaparecimento da pastagem, estimada com o auxílio da técnica do triplo emparelhamento e calculada pela equação de Moraes (1991), quantificou a forragem que foi cortada e consumida pelo pastejo dos cordeiros, incluindo, também, as porções de tecidos das plantas da pastagem consumidas por organismos decompositores. Segundo Walter \& Evans (1979), a taxa de desaparecimento de forragem, em alguns casos, pode proporcionar estimativas acuradas do consumo de bovinos em pastejo. Todavia, Linehan \& Lowe (1946), usando gaiolas e com equações diferentes da utilizada neste experimento, afirmam que o consumo de forragem pode ser superestimado quando baseado na taxa de desaparecimento da pastagem.

Os níveis de RMS não tiveram efeito sobre a participação do trevo-branco. A participação percentual da leguminosa variou de $1,1 \%$ a $5,3 \%$. Tal fato foi causado pelo alto nível de RMS/ha na fase pré-experimental, que causou sombreamento das plantas de azevém sob as de trevo-branco, e ao baixo nível de $\mathrm{P}$ no solo, em todas as unidades experimentais. Com relação à percentagem de $\mathrm{PB}$, não houve efeito dos tratamentos sobre esta variável. Isto poderia estar relacionado ao fato de as plantas, mesmo com proporções consideráveis de tecidos senescentes, ainda apresentarem bons teores de PB, conforme relatou Moraes (1991).

TABELA2. Taxa de desaparecimento da pastagem, participação percentual do trevo branco, percentagem de proteína bruta, número de animais.dia/ha e carga animal na pastagem de azevém e trevo-branco submetida a níveis de resíduo de forragem.

\begin{tabular}{cccccc}
\hline $\begin{array}{c}\text { Resíduos de forragem } \\
\text { (kg MS/ha) }\end{array}$ & $\begin{array}{c}\text { Taxa de } \\
\text { desaparecimento } \\
\text { (kg MS/ha/dia) }\end{array}$ & $\begin{array}{c}\text { Trevo-branco } \\
(\%)\end{array}$ & $\begin{array}{c}\text { Proteína bruta } \\
(\%)\end{array}$ & $\begin{array}{c}\text { Animais } \\
\text { dia/ha } \\
\left(\mathrm{n}^{\mathbf{0}}\right)\end{array}$ & $\begin{array}{c}\text { Carga animal } \\
(\mathrm{kg} / \mathrm{ha})\end{array}$ \\
\hline 1.119 & 82,6 & 3,0 & 13,2 & 4.215 & 1.117 \\
1.320 & 91,2 & 2,7 & 13,0 & 4.052 & 1.119 \\
1.477 & 104,0 & 1,1 & 11,3 & 4.670 & 1.181 \\
1.695 & 79,1 & 3,1 & 13,4 & 4.652 & 1.265 \\
2.146 & 70,4 & 5,3 & 13,5 & 3.788 & 1.016 \\
2.166 & 101,1 & 3,5 & 14,8 & 4.637 & 1.255 \\
2.410 & 52,9 & 2,6 & 9,9 & 3.685 & 1.079 \\
2.483 & 75,7 & 3,2 & 14,3 & 4.059 & 1.190 \\
\hline
\end{tabular}


A falta de significância entre carga animal e número de animais.dia/ha com os RMS foi resultante da semelhança na produção de MS. Nos estudos de pastejo com utilização da técnica das lotações variáveis, a carga animal e por conseguinte o número de animais.dia/ha são ajustados com base nas estimativas da taxa de acúmulo de MS, que determina a produção de MS. Como essas duas variáveis não diferiram neste experimento, igual comportamento foi constatado para a carga animal e número de animais.dia/ha. A primeira oscilou de 1.016 a $1.265 \mathrm{~kg}$ de cordeiros/ha, e a segunda variou de 3.685 no RMS com $2.410 \mathrm{~kg}$ de $\mathrm{MS} / \mathrm{ha}$ a 4.670 no RMS com $1.477 \mathrm{~kg}$ de MS/ha.

Com base nos resultados aqui apresentados, ficou fundamentado que as pastagens de inverno devem apresentar altos níveis de RMS e altas disponibilidades de forragem quando utilizadas com cordeiros oriundos de campo nativo, e portanto criados com baixos níveis alimentares.

\section{CONCLUSÃO}

Com cordeiros, o manejo da pastagem deve ser feito em níveis próximos a $2.400 \mathrm{~kg}$ de $\mathrm{MS} / \mathrm{ha}$.

\section{REFERÊNCIAS}

ASSOCIATION OF OFFICIAL AGRICULTURAL CHEMISTS. Official Methods of Analysis. 14.ed. Washington, 1984. 1141p.

BLASER, R.E. Stobbs Memorial Lecture 1981. Integrated pasture and animal management. Tropical Grasslands, Brisbane, v.16, n.1, p.9-24, Mar. 1982.

BURNS, J.C.; LIPPKE, H.; FISHER, D.S. The relationship of herbage mass and characteristics to animal responses in grazing experiments. In: MARTEN, G.C. (Ed.). Grazing research: Design methodology, and analysis. Madison: Crop Science Society of America/American Society of Agronomy, 1989. Ch.2, p.7-19.

CAMPBELL, A.G. Grazed pastures parameters. I. Pasture dry-matter production and availability in a stocking rate and grazing management experiment with dairy cows. Journal of Agricultural Science, Cambridge, v.67, n.2, p.211-216, Oct. 1966.
FIGUEIRÓ, P.R.P. Efeito do cruzamento da raça Hampshire Down e Romney Marsh na produção de cordeiros para abate. Revista do Centro de Ciências Rurais, Santa Maria, v.9, n.4, p.421-428, dez. 1979.

GARDNER, A.L. Medição dos atributos das pastagens em experimentos de pastejo. In: GARDNER, A.L. "Técnicas de pesquisa em pastagens e aplicabilidade de resultados em sistemas de produção". Brasília: Embrapa, 1986. p.113-140.

GEENTY, K.G. Pasture feeding for high lamb growth during the lactation period. In: FAMILTON, A.S. (Ed.). Lamb growth farmers handbook. Canterbury: Lincoln College, 1983. Ch.3., p.23-27.

GIBB, M.J.; TREACHER, T.T. The effect of ewe body condition at lambing on the performance of ewes and their lambs at pasture. Journal of Agricultural Science, Cambridge, v.95, n.3, p.631-640, Dec. 1980.

GIBB, M.J.; TREACHER, T.T. The effect of herbage allowance on herbage intake and performance of lambs grazing perennial ryegrass and redclover swards. Journal of Agricultural Science, Cambridge, v.86, n.2, p.355-365, Apr. 1976.

HENDRICKSEN, R.; MINSON, D.J. The feed intake and grazing behaviour of cattle grazing a crop of Lablab purpureus cv. Rongai. Journal of Agricultural Science, Cambridge, v.95, n.3, p.547-554, Dec. 1980.

LINEHAN, P.A.; LOWE, J. The output of pasture and its measurement. Journal of the British Grassland Society, Aberystwyth, v.1, n.1, p.7-35, Mar. 1946.

MAXWELL, T.I. Factors affecting the growth and utilization of sown grasslands for sheep production. In: HARESIGN, W. (Ed.). Sheep production. London: Butterworths, 1983. Ch.10, p.187-205.

MOOJEN, E.L. Dinâmica e potencial produtivo de uma pastagem nativa do Rio Grande do Sul submetida a pressões de pastejo, épocas de diferimento e níveis de adubação. Porto Alegre: UFRGS, 1991. 172p. Tese de Doutorado.

MORAES, A. Produtividade animal e dinâmica de uma pastagem de pangola (Digitaria decubens stent), azevém (Lolium multiflorum Lam.) e trevo branco (Trifolium repens $\mathrm{L}$.) submetida a diferentes pressões de pastejo. Porto Alegre: UFRGS, 1991. 200p. Tese de Doutorado. 
MOTT, G.O.; LUCAS, H.L. The design, conduct and interpretation of grazing trials on cultivated and improved pastures. In: INTERNATIONAL GRASSLAND CONGRESS, 6., 1952 , Pennsylvania. Proceedings... Pennsylvania: State College Press, 1952. p.1380-1385.

ORR，R.J.; PARSONS，A.J.; PENNING，P.D.; TREACHER, T.T. Sward composition, animal performance and the potential production of grass/white clover swards continuously stocked with sheep. Grass and Forage Science, Oxford, v.45, n.3, p.325-336, Sept. 1990.

PENNING, P.D.; HOOPER, G.E.; TREACHER, T.T. The effect of herbage allowance on intake and performance of ewes suckling twin lambs. Grass and Forage Science, Oxford, v.41, n.3, p.199-208, Sept. 1986.

PENNING, P.D.; PARSONS, A.J.; ORR, R.J.; TREACHER, T.T. Intake and behaviour responses by sheep to changes in sward characteristics under continuous stocking. Grass and Forage Science, Oxford, v.46, n.1, p.15-28, Mar. 1991.

PIRES, M.B.G.; FREITAS, E.A.G.; TRINDADE, D.S Estabelecimento de um sistema de digestibilidade ïn vitro no Laboratório da Equipe de Pesquisas em Nutrição Animal da Secretaria da Agricultura. Anuário Técnico do IPZFO, Porto Alegre, v.6, p.345-385, 1979.

QUADROS, F.L.F. de; MARASCHIN, G.E. Desempenho animal em misturas de espécies forrageiras de estação fria. Pesquisa Agropecuária Brasileira, Brasília, v.22, n.5, p.535-541, maio 1987.
RATTRAY, P.V.; THOMPSON, K.F.; HAWKER, H.; SUMNER, R.M.W. Pastures for sheep production. In: NICOL, A.M. (Ed.). Feeding livestock on pasture. Hamilton: New Zealand Society of Animal Production, 1987. Ch.7, p.89-103.

SAS INSTITUTE. User's Guide. Version, 5.ed. Cary, NC: SAS Institute Inc., 1985. 956p.

SHEATH, G.W.; RATTRAY, P.V.; SMEATON, D.C. Influence of pasture quantity and quality on intake and production of sheep. In: HORN, F.P.; HODGSON, J.; MOTT, J.J.; BROUGHAM, R.W. (Eds.). Grazing-lands research at the plant-animal interface. Morrilton:Winrock International Institute for Agricultural Development, 1987. Ch.3, p.33-43

SIQUEIRA, E.R. de; OSÓRIO, J.C. da S.; GUERREIRO, J.L.V.; JARDIM, P.O. da C. Desempenho de cordeiros machos e fêmeas da raça Ideal e cruzas Texel x Ideal, criados em pastagem nativa. Pesquisa Agropecuária Brasileira, Brasília, v.19, n.12, p.1523-1528, dez. 1984.

TILLEY, J.M.A.; TERRY, R.A. A two-stage technique for in vitro digestion of forage crop. Journal of the British Grassland Society, Hurley, v.18, n.2, p.104-111, Feb. 1963.

WALTER, R.J.K.; EVANS, E.M. Evaluation of a sward sampling technique for estimating herbage intake by grazing sheep. Grass and Forage Science, Oxford, v.34, n.1, p.37-45, Mar. 1979.

WILM, H.G.; COSTELO, O.F.; KLIPPE, G.E. Estimating forage yield by the double sampling method. Journal of the American Society of Agronomy, New York, v.36, n.1, p.194-203, Sept. 1944. 